\title{
CHIASMA FREQUENCY AND LONGEVITY IN LOLIUM MULTIFLORUM
}

\author{
J. G. CROWLEY* \\ Department of Agricultural Botany, University College of Wales, Aberystwyth
}

Received 7.iv.68

\section{IntRoduction}

IN Lolium perenne chiasma frequencies are closely correlated with the longevity of populations (Rees and Ahmad, 1962). Populations of annual habit have higher chiasma frequencies than more " perennial " populations. Results from rye (Sun and Rees, 1964) agreed closely with those from Lolium. It was suggested that this relationship between chiasma frequency and longevity might well be of adaptive significance. The present work examines chiasma frequency variation in relation to longevity in another Lolium species, viz., Lolium multiflorum. This species is especially suitable for such an investigation because many varieties are available which differ widely in the duration of their life-cycles.

\section{Materials AND methods}

Seven varieties of Lolium multiflorum were compared (table 1). They range from strictly annual to relatively perennial.

First, 50 plants of each variety were sown in boxes on 31st January 1967 and the seedlings subsequently planted in the field in a randomised block experiment with 5 replications of 10 plants each. Chiasma frequencies were scored in 20 pollen mother cells (p.m.c.) in a random sample of 20 plants from each variety ( 4 plants from each replicate). The chiasma frequency was expressed as the average per cell.

A second sowing was carried out on 16th April. It consisted of the same 7 varieties but, in this case, each variety was represented by 60 plants sown as a randomised block experiment with 3 replicates. The proportion of plants having flowered in each variety was estimated at the close of the season (September).

There was no significant variation between replicates in respect either of flowering or chiasma frequency. For this reason the data in the tables and figures below are pooled over replicates.

\section{Results}

\section{(a) The "perenniality index"}

Cooper (1960) has shown that the proportion of plants flowering in the first season following a late spring sowing is a good index of the "perenniality" of a population. The more " perennial " the population the lower is the proportion of plants flowering - and vice versa. The proportion for each variety, the "perenniality index" (P.I.), is given in table 1. These

* Present address: The Agricultural Institute, Oakpark, Carlow, Ireland. 
P.I. values are estimated from the April-sown plants. Plants derived from the January sown seed all fowered-as indeed was to be expected.

The P.I. values vary considerably between varieties. The differences are significant $(P=<0.001)$.

\section{(b) Chiasma frequency and perenniality}

It is particularly important to emphasise that the chiasma frequencies were estimated in all cases from populations sown in January. It will be recalled that following such early sowing all plants flowered in all populations Consequently the chiasma frequencies, even in the more "perennial" populations, are from a random sample of plants and not simply those which flowered in the first year, such as would be the case after a late sowing.

TABLE 1

The perenniality index (P.I.) along with the mean chiasma frequency in the seven populations

$\begin{array}{lcc}\quad \text { Variety } & \begin{array}{c}\text { Perenniality } \\ \text { index } \\ (P . I .)\end{array} & \begin{array}{c}\text { Chiasma } \\ \text { frequency } \\ \text { per } p . m . c .\end{array} \\ \text { 1. Portuguese I.S.E.S. } & 90 \cdot 0 & 13 \cdot 33 \\ \text { 2. Witala } & 56 \cdot 0 & 12 \cdot 70 \\ \text { 3. Irish Commercial } & 45 \cdot 0 & 12 \cdot 57 \\ \text { 4. C.I.V. } & 34 \cdot 0 & 12 \cdot 42 \\ \text { 5. Dasas } & 43 \cdot 1 & 12 \cdot 22 \\ \text { 6. S.22 } & 32 \cdot 1 & 12 \cdot 18 \\ \text { 7. Tiara } & 18 \cdot 4 & 12 \cdot 01\end{array}$

This, of course, rules out any bias that might have been due to a variation in chiasma frequency between plants which flowered in the first year as compared with those not flowering until the second year.

The chiasma frequencies are given in table 1. Differences between populations are significant $(\mathrm{P}=<0.001)$.

In fig. 1 the mean chiasma frequencies of populations are plotted against their P.I. values. From this graph it is clear that the chiasma frequencies increase with increasing P.I. values. In other words, the more " annual" the population the higher its chiasma frequency. A regression analysis of variance confirms the relationship. The regression is positive and highly significant $(P=<0.001)$. The results agree completely with those described by Rees and Ahmad in L. perenne.

\section{(c) The question of pleiotropy}

Before one concludes, as did Rees and Ahmad (loc. cit.) and Sun and Rees (loc. cit.), that the variation in chiasma frequency is adaptive and determined by selection in relation to the longevity of a population it is necessary to inquire into another possible cause of the correlation between the two characters. The alternative possibility is that the physiology of plants which flower readily after spring sowing is such that these plants also have high chiasma frequencies at meiosis. Put in genetic terms, this would mean that genes permitting flowering after late sowing have a pleiotropic action 
that determines a high chiasma frequency. What evidence there is suggests the contrary, as is explained below.

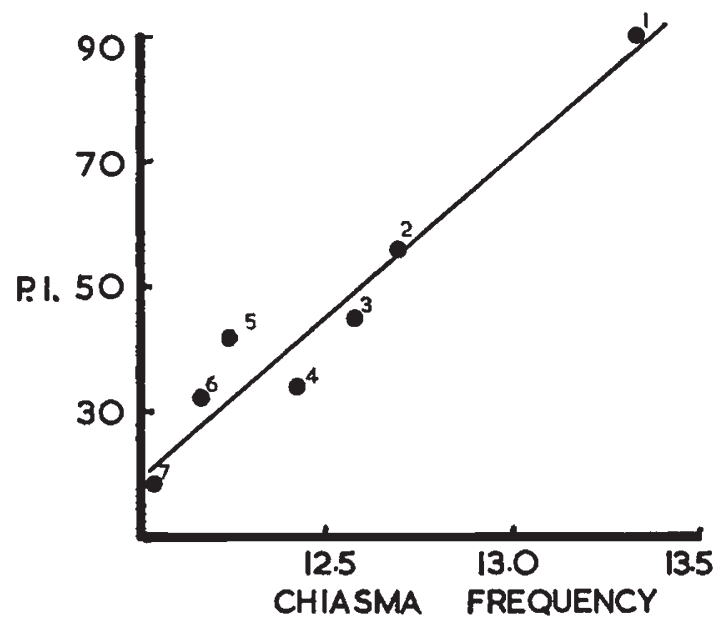

FIG. 1.-The perenniality indices (in angular values) plotted against the average plant chiasma frequencies in the 7 varieties. Numbers relate to the order of families in table 1 .

The high flowering potential of plants in short-lived populations of L. multiflorum is reflected not only by their readiness to flower after late spring sowing but also, as one might expect, by their flowering early in the season

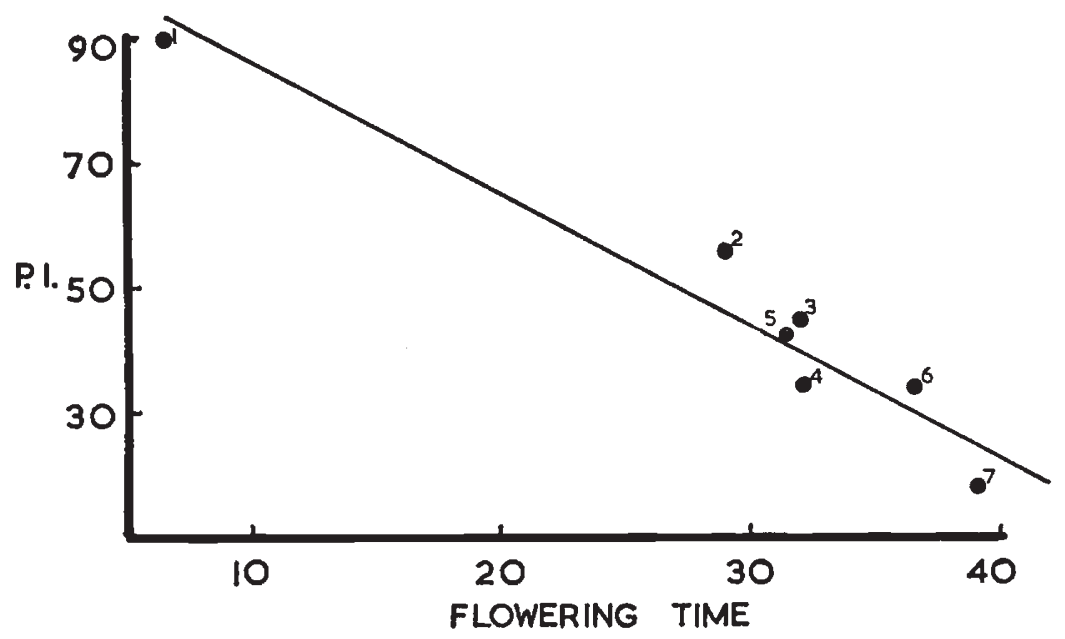

Fig. 2.-The perenniality indices (in angular values) plotted against the flowering time in the 7 varieties. Numbers as in fig. 1 .

as compared with plants from longer-lived populations. This is clearly illustrated in fig. 2 , in which the average flowering time is plotted against the P.I. for each of the 7 varieties. The regression of flowering time on P.I. is negative and highly significant $(P=<0.001)$. It follows that if the chiasma frequency variation is due to a pleiotropy of genes controlling flowering we 


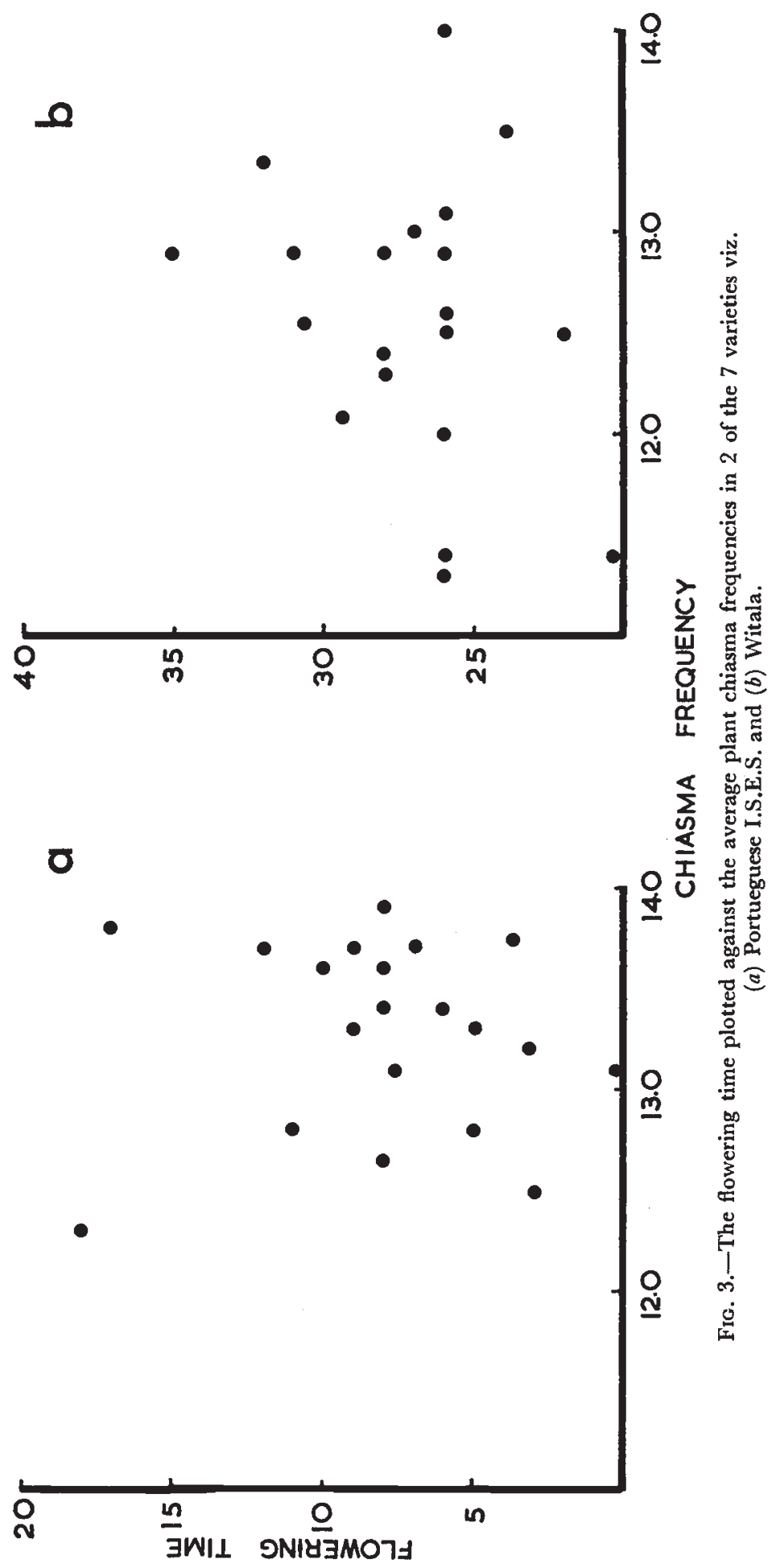


should expect, within populations, a correlation between plant flowering time and chiasma frequency-in the present context a negative correlation. There is no evidence whatsoever of such a correlation (fig. 3). We must conclude, therefore, that the chiasma frequency variation between the varieties is not the consequence of a pleiotropy by genes which determine differences in flowering.

\section{(d) Chiasma frequency and fertility}

It could also be argued that the higher chiasma frequencies in the shorter-lived populations could simply be the outcome of a selection for high fertility because a high seed set clearly has high priority where propagation depends largely on re-seeding. Plants with very low chiasma frequencies leading to failure of chromosome pairing and to univalents would be at a severe disadvantage and, therefore, liable to be eliminated by selection.

TABLE 2

The between plant variance for chiasma frequency and for flowering time in each of the seven populations

\begin{tabular}{lccc}
\multicolumn{1}{c}{ Variety } & & $\begin{array}{c}\text { Chiasma } \\
\text { frequency } \\
\text { variance }\end{array}$ & $\begin{array}{c}\text { Flowering } \\
\text { time } \\
\text { variance }\end{array}$ \\
1. Portuguese I.S.E.S. & $(90.0)$ & 0.30 & 10.86 \\
2. Witala & $(56.0)$ & 0.43 & 15.69 \\
3. Irish Commercial & $(45.0)$ & 0.45 & 22.71 \\
4. C.I.V. & $(34.0)$ & 0.45 & 21.00 \\
5. Dasas & $(43.1)$ & 0.62 & 21.80 \\
6. S.22 & $(32.1)$ & 0.51 & 18.38 \\
7. Tiara & $(18.4)$ & 0.54 & 29.44
\end{tabular}

Flowering time was scored as the number of days to heading after 12 th May. P.I. values in brackets.

No such explanation, however, will adequately account for the chiasma frequency variation between the present populations because univalents were absent even in the scores for the more perennial populations with the lowest chiasma frequencies.

\section{Ghiasmata and variability}

As has previously been pointed out by Rees and Ahmad (loc. cit.), the higher chiasma frequencies in the shorter-lived populations is somewhat unexpected. In short-lived populations one expects, on theoretical grounds, to find mechanisms restricting variability (see for example Darlington, 1939; Mather, 1943), and there is much evidence to support this general contention. Thus annuals are more often inbreeding and have fewer chromosomes than perennials (Grant, 1958; Stebbins, 1958). Both features serve to restrict the variability of populations, the first by creating and maintaining homozygosity, and the second by reducing recombination within the chromosome complement. While it is, therefore, undeniable that a restriction on variability is the general rule among short-lived populations it would, at the same time, be wrong to suppose that all components of the genetic system of such populations would necessarily work in a complementary, restrictive fashion. This point has been emphasised by both Grant (loc. cit.) and by Stebbins 
(loc. cit.). For example, they both cite the common occurrence of higher chiasma frequencies in inbreeders as compared with related outbreeders.
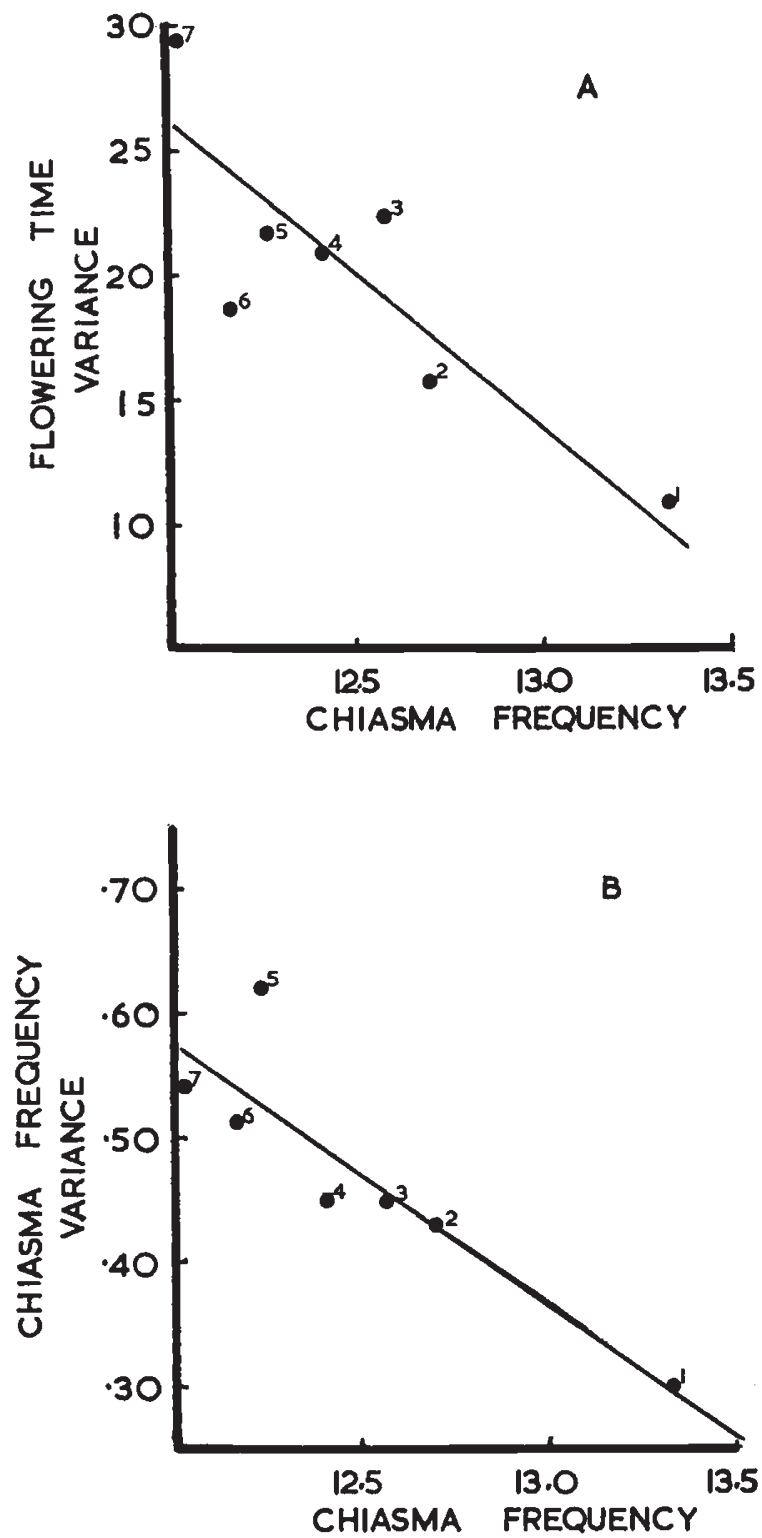

Fig. 4.-The between plant variances for two characters $(a)$ flowering time and $(b)$ chiasma frequency plotted against the average plant chiasma frequencies in the 7 varieties. Numbers as in fig. 1.

Here the one component, in the form of a high chisama frequency, would serve to increase the variability whereas the other, the inbreeding, has the opposite effect. They interpret such apparently contradictory situations in 
terms of a compromise whereby the extreme uniformity which results from inbreeding is compensated by a high degree of recombination (and of variability released) following rare but perhaps inevitable outcrossing in normally inbred populations. Rees and Ahmad (loc. cit.) and Jones and Rees (1966) have produced evidence indicating that the high chiasma frequencies in short-lived Lolium perenne populations serve also to compensate for a lower potential variability in these populations as compared with longer-lived populations.

An attempt has been made to find out whether the higher chiasma frequencies of the shorter-lived Lolium multiflorum populations investigated in the present work may also compensate for a lower potential variability. Only detailed selection experiments can yield reliable measures of the potential genetic variability of a population, but a simple index, for comparative purposes, was obtained for the seven Lolium multiflorum populations by calculating the between-plant variance for each of two characters in each population. The characters were flowering time and the mean plant chiasma frequency (table 2). The reasonable assumption is made that the higher the variance the greater the potential variability. On this basis it is clear from the table that the more short-lived the population the lower the potential variability. As in Lolium perenne, their higher chiasma frequencies may well compensate for the lower potential genetic variability. Indeed, when the variances for the two characters are plotted against the chiasma frequencies of the 7 populations it will be seen that, in both cases, there is a convincing negative correlation (figs. $4 a$ and $b$ ). The regressions are significant $(\mathrm{P}=<0.05$ and $<0.01$, respectively). In so far as these variances reflect directly the potential variability, there is in $L$. multiflorum, as in $L$. perenne, a compensating increase in chiasma frequency with decreasing potential variability which, in turn, is closely dependent upon logevity.

It needs to be emphasised that all of the plants investigated are representative not of natural populations but of bred varieties. Consequently, while it is both reasonable and feasible to conceive of the chiasma frequency variation between these populations as the adaptive consequences of selection it is not clear whether such variation is the result of selection during breeding or is a relic of the selection imposed upon the natural populations from which the varieties were originally derived. The latter appears the more likely. For one thing it is difficult to visualise such a subtle change in the genetic system of a population as a consequence of a relatively short-term breeding programme.

\section{Summary}

1. In Lolium multiflorum chiasma frequency is correlated with longevity. The chiasma frequencies are higher in short-lived than in more " perennial" populations.

2. There is evidence that the higher chiasma frequencies in the shortlived populations may compensate for their relatively low potential genetic variability.

Acknowledgments.-The author expresses his gratitude to $\mathrm{Dr} \mathrm{H}$. Rees for his advice and encouragement during the course of the experiment and in the preparation of the manuscript. I am indebted also to Dr A. Durrant of this department and to Dr J. P. Cooper of the Welsh Plant Breeding Station, Aberystwyth, for helpful discussion.

The author acknowledges with gratitude the receipt of a two-year leave of absence from the Agricultural Institute, Ireland. 


\section{REFERENCES}

COOPER, J. P. 1960. Short-day and low-temperature induction in Lolium. Anns. Bot., 24, $232-246$.

Darlington, C. D. 1939. The Evolution of Genetic Systems. Cambridge University Press. GRANT, v. 1958. Regulation of recombination in plants. Cold Spring Harb. Symp. Quant. Biol., 23, 337.

MATHER, K. 1943. Polygenic inheritance and natural selection. Biol. Rev., 18, 32-64.

REES, H., AND AHMAD, x. 1963. Chiasma frequencies in Lolium populations. Evolution, 17, 575.

STEBBINS, G. K. 1958. Longevity, habit and release of genetic variability in higher plants. Cold Spring Harb. Symp. Quant. Biol., 23, 337.

SUN, S., AND REES, H. 1964. Genotypic control of chromosome behaviour in rye. VII. Unadaptive heterozygotes. Heredity, 19, 357. 